\title{
Anti-TNF and pouch surgery for ulcerative colitis: the ones who blame for more complications?
}

\author{
G. Bislenghi*, M. Ferrante**, A. D’Hoore* \\ *Department of Abdominal Surgery, University Hospitals Leuven, KU Leuven, Leuven, Belgium. \\ **Department of Gastroenterology and Hepatology, University Hospitals Leuven, KU Leuven, Leuven, \\ Belgium.
}

\begin{abstract}
Since the approval in 2005 of anti-TNF drugs for the treatment of ulcerative colitis, concerns have been raised about the potential detrimental effect of these agents on postoperative infectious complications related to pouch surgery. Data on this topic are controversial and mostly derived from retrospective underpowered cohort studies largely affected by relevant bias. Three meta-analyses have been published with contradictory results. Moreover, the correlation between serum levels of infliximab at the time of surgery and the occurrence of septic postoperative complication is far to be proven and remains an answered research question.

The construction of an ileal pouch-anal anastomosis (IPAA) as first surgical step in patients with ulcerative colitis (UC) refractory to medical treatment seems to be associated with an increased risk of septic complications. Population-based data from the United States show a shift towards stage surgery for patients with refractory $U C$ as consequence of the widespread use of biological agents and the increased tendency to consider surgery as ultimate resort (step-up approach). In this setting, the classic 3-stage procedure (ileoanal pouch and diversion ileostomy after initial total colectomy) together with the modified 2-stage approach (ileoanal pouch without diversion ileostomy after initial total colectomy) are both effective options. Whether or not a diversion ileostomy could prevent pouch complications at the time of the pouch construction during the second stage of surgery is still matter of debate. Emerging data seem to claim for increased risk of small bowel obstructions related to the presence of a stoma without proven effect on the prevention of anastomotic leak.
\end{abstract}

\section{Background}

Since their approval in 2005 by the U.S. Food and Drug Administration for the treatment of inflammatory bowel disease, the use of anti-tumor necrosis factor (anti-TNF) agents in patients with moderate-to-severe ulcerative colitis (UC) has gained an ever-expanding role. (1) Three main agents, adalimumab, golimumab, and infliximab (IFX) are currently used in the treatment algorithms for UC as an effective option for patients with steroid-dependent active disease or who have failed conventional therapy. Furthermore, infliximab is an effective salvage therapy in patients with severe UC refractory to intravenous steroids. (2)

Biological therapy has reduced the need for emergency and urgency colectomy. However, up to half patients so rescued fail to have sustained remission and ultimately will require interval colectomy due to disease recurrence, progressive side effects of medical treatment, or, in the medium-term, dysplasia or malignancy in the colon. (3) Despite improving medical therapy, 10 to $30 \%$ of patients with UC will need surgery at some point. Younger patients present a double risk of losing response to medical treatment. (4-7) Furthermore, anti-TNF agents seem to increase the risk of opportunistic infections (bacterial and mycotic) and may potentially delay wound healing. (8) The risk of infections associated with the use of anti-TNF agents can be thought of in terms of bacterial infections such as upper respiratory tract and urinary tract infections, rare but potentially life-threatening opportunistic infections, reactivation of latent tuberculosis, viral infections 
(such as hepatitis B) and vaccine preventable disease. (9-12) Given the increasing amount of UC patients exposed to anti-TNF during the course of their disease, concerns have been raised regarding the safety of anti-TNF agents in the setting of abdominal surgery. Restorative proctocolectomy with ileal pouch-anal anastomosis (IPAA) is the surgical treatment of choice in UC, since it maintains intestinal continuity and avoids the need for a permanent stoma. (13) However, complications as anastomotic leak, pelvic abscess, wound infection, bleeding and small bowel obstruction (SBO) are well documented. (14) Over the years, a plethora of studies and meta-analyses have tried to answer the question whether the perioperative use of anti-TNF agents promotes the o of postoperative infectious complications after restorative proctocolectomy in UC patients or not. Unfortunately, data provided conflicting results.

With this in mind, this narrative review has the purpose to describe all relevant data available, to give an insightful discussion from a surgical perspective over the real impact of anti-TNF agents in outcomes after pouch surgery for ulcerative colitis.

\section{Review}

\section{- Anti-TNF, surgery and ulcerative colitis}

TNF is an important cytokine implicated in regulating wound healing and protection against infections. It plays a key role as mediator of neutrophil chemotaxis and adhesion during the initial phases of inflammation. (15) As already mentioned, anti TNF therapy has been associated to higher rates of opportunistic infections (both bacterial and mycotic) such as urinary and respiratory tract infections, tuberculosis reactivation, candidiasis and histoplasmosis among others. (8) Additionally, in vivo animal studies have shown a higher ingrowth rate of granulation tissue and increased wound-breaking strength when TNF was locally applied to incised or traumatic wounds, providing evidence that TNF antagonists might contribute to impair wound healing. $(16,17)$ On the other hand, some other studies suggest a possible protective role of anti-TNF agents on wound healing. After local administration of TNF, the ingrowth rate of granulation tissue in histological specimen was significantly lower after 7 days compared to control. Salomon et al found a possible explanation in the decreased synthesis of collagen, the basic protein element in healing, induced by TNF. When exogenous TNF was added to wounds it reduced the expression of the gene for type 1 collagen and decreased the force required to disrupt the wound. $(18,19)$

Nowadays, restorative proctocolectomy with ileal pouch-anal (IPAA) anastomosis is indicated for UC refractory to medical therapy or when dysplasia or cancer occurs. Laparoscopic pouch surgery is a feasible and safe option as shown in several reports that have investigated the superiority, in terms of both shortand long-term outcomes, of the minimally invasive approach. (20) IPAA can be performed with either mucosectomy of the rectal mucosa, with a hand-sewn anastomosis to the dentate line, or with a doublestapling technique. The first technique has clearly the advantage to remove entirely the rectal mucosa and to eliminate the risk of malignant transformation. In the second, a rectal cuff of $3 \mathrm{~cm}$ length is preserved. This guarantees better functional results despite a higher risk of cuffitis. (21) As ultimate evolution of minimally invasive surgery, our group proposed in 2016 a single-stapling technique combining single port laparoscopy (SILS) through the ileostomy site and a trans-anal approach to perform the proctectomy and the anastomosis (ta-IPAA). (22) Regardless of the technique used, IPAA remains a technically demanding procedure with an overall 30 -day complication rate of $30 \%$ to $40 \%$ and a specific pelvic sepsis rate of about $10 \%$. $(20,23)$ Overall pouch failure is reported to range from $0.5 \%$ to $24 \%$. The frequency increases over time, reaching the highest values during long-term follow up. (2) Pouch failure is generally defined as pouch excision or permanent diversion due to debilitating pouch dysfunction or to failure to correct pouch complications. (24) With regard to this, several studies have demonstrated the relation between the occurrence of postoperatively pelvis sepsis and long-term pouch failure after IPAA. $(25,26)$ 
UC patients may be prone to develop more postoperative infectious complications given the prolonged exposure to extensive medical therapy (steroids, immunomodulators, biologics), leaving these patients in worse conditions at the time of surgery. The use of anti-TNF drugs might be seen as a surrogate for more severe disease and sicker UC patients. The impact of anti TNF agents on postoperative outcomes after pouch surgery is largely controversial. Results are derived from retrospective, small studies with remarkable bias related to surgical technique, timing of surgery, interval between last administration of biologics and surgery, heterogeneity of study populations with regard to type and duration of exposure to preoperative medications, concomitant therapy regimens, comorbidities, disease severity, preoperative nutritional and general conditions. Given the complexity of surgery, dependent on the severity of the disease and the use of preoperative steroids and/or biologics, a stage surgical approach is usually performed when operating on patients with refractory UC. (27) This in the attempt to minimize the amount of postoperative complications. An overview of the different surgical strategies is given in Table 1.

\section{- Cohort studies}

Major concerns over the association between anti TNF agents and postoperative infectious complications raised after the publications of two retrospective studies from Mayo and Cleveland clinic, respectively in 2007 and 2008. $(28,29)$ Colleagues from Mayo clinic published data on 301 UC patients, of whom 47 patients had ever been treated with infliximab. They found an increased risk for pouch-specific and overall infectious complications after 2- or 3-stage restorative proctocolectomy in the 47 patients treated with IFX compared to the control group. Important criticisms of this study are the off-label use of IFX prior to 2005 suggesting a higher rate of sicker patients potentially prone to develop septic complications after IPAA, a lack of homogeneity between the IFX and non-IFX groups related to age, severity of colitis, and concomitant use of other medications such as steroids and immunomodulators. Furthermore, more than half of the patients received a last dose of IFX more than 8 weeks prior to the index surgery. No difference was found in terms of infectious complications when these patients were compared to those who had been on IFX within 8 weeks of IPAA. A similar conclusion was reached by investigators from Cleveland clinic in a more homogeneous cohort of IPAA patients of whom 85 preoperatively exposed to IFX, with a median time between last IFX administration and surgery (2- or 3-stage proctocolectomy) of 13.5 weeks. They concluded that IFX increases the risk of overall and infectious postoperative complications after restorative proctocolectomy and altered the surgical approach to UC in their case series. At this regard, an independent association of 3-stage proctocolectomy with IFX was shown. Reported leak rate of IPAA was higher in the IFX group. They concluded that previous (ever) IFX should led the patients to a 3-stage procedure, due to the high risk of poor outcomes. These findings are consistent with what has been shown by Eshuis et al in 2012. Increased rate of pelvic sepsis was reported in 21 patients treated with IFX (median interval between last administration of IFX and surgery of 7 months) who received IPAA as first surgical step. No difference was observed when pouch construction was postponed to a second stage of surgery. (30)

By contrast, other subsequent studies including a mixed cohorts of patients undergoing both resections and IPAA, failed to find a correlation between anti-TNF agents and postoperative infectious complications. (3134) In 2009, our group analyzed retrospectively data from 22 patients recently exposed to IFX and compared to those of a control group of 122 patients. No difference in terms of concomitant medications and disease extent was present at the time of the first surgery. Patients in the IFX group less often underwent a proctocolectomy without a defunctioning ileostomy (one-stage procedure). In none of the subgroup analyses IFX was found to be a risk factor for postoperative infectious complications. By contrast, corticosteroids and one-stage restorative proctocolectomy resulted to be independent predictors of short-term postoperative infectious complications. (35) Our results are broadly in line with those coming from one of the most relevant centre for the treatment of inflammatory bowel disease in North America, which have been recently published. (36) Retrieving data from 758 patients with UC/IPAA, of whom 196 ever treated with IFX, no increase in the postoperative IPAA leak rate was found in the IFX group. Even when a traditional 2-stage 
procedure (37 IFX vs 186 non-IFX patients) was separately analyzed, no difference between groups could be assessed.

\section{- Meta-analyses}

In literature, three meta-analyses have been written on this topic. (37-39) In a first attempt in 2010, Yang et al published results supporting the association between anti-TNF and short-term postoperative complications. These conclusions were largely influenced by the results of the previous mentioned cohort studies from Mayo and Cleveland clinic, both supporting the role of anti-TNF in increasing postoperative infective complications. $(28,29)$ The same authors implemented their analyses two years later, including 13 studies and 2900 patients with more consistent data and concluded, in contrast with their previous statement, that recent or ever used IFX was an independent factor increasing the rate of total postoperative complications $(O R=1.80)$, but not significant if only looking at inflammatory complications. This might be explained with the inclusion of more trials published in the interval period between the two meta-analyses which failed to find a correlation between anti-TNF and postoperative complications. As effect of this, the overall evaluation switched to absence of association between IFX and postoperative infectious complications. Unfortunately, these meta-analyses presented many limitations related to the quality and different settings (cohort, case-control, registry-based studies) of the selected studies, different definitions of IFX exposure (any previous exposure vs within 8 of 12 weeks of primary surgery), different definitions of early-infectious, non-infectious and total postoperative complications. Furthermore, several confounding factors such as disease severity at primary surgery, concomitant therapy, type of IPAA surgery, comorbidity, general and nutritional status at the time of surgery, surgeon experience were not adequately considered in the analysis. Despite these limitations, these two meta-analyses coming from the same group, providing diametrically opposite conclusions, are emblematic of the controversy on this topic.

A well-conducted meta-analysis, targeted at identifying the effect of biologics on complications after proctocolectomy and IPAA as first surgery, included 7 studies with more than 600 patients and provided interesting conclusions. From the pooled analysis emerged that patients who received biologics presented a higher risk of IPAA-related complications, affecting 1 of 5 patients undergoing surgery in this setting. The risk was not attenuated by the presence of a diversion ileostomy, with IPAA-related complications after ileostomy closure occurring in $25 \%$ of the cases. No increased risk for both overall and infectious complications was found if the analysis was conducted regardless of the type of surgery performed. Preoperative IFX use did not increase the risk of early postoperative complications in patients with UC undergoing abdominal surgery, but construction of the pouch should be postponed to a second stage. Likewise, the ECCO consensus on surgery for UC recommended that surgeons avoid to perform proctocolectomy and IPAA at once as first surgery in anti-TNF treated patients. (2)

\section{- Unanswered research questions}

Concerning the interval between last administration of anti-TNF drugs and surgery, most of the studies assumed 12 weeks as ideal cut-off for IFX. This interval time is derived from the well documented disappearance of anti-IFX antibodies at 12 weeks after the last administration of IFX. (40) However, some authors contest this statement and suggest that a lower threshold might be chosen because of the variability in the half-life of IFX with a wide range from 7 to 18 days. $(41,42)$ Some studies have tried to identify a link between the occurrence of postoperative complications and the preoperatively serum levels of IFX. $(43,44)$. In a large series of 473 mixed Crohn disease (CD) and UC patients, data of 195 patients with documented preoperative exposure to anti-TNF agents (IFX and/or adalimumab) within 180 days from the date of surgery were carefully reviewed. A time-dependent analysis on three subgroups (exposure to anti-TNF within 14 days, within 15 and 30 days and within 31 and 180 days of the surgery) was performed. No difference was found in terms of infectious complications rate between the three groups. A subgroup analysis was 
conducted on a small subset of patients with UC exposed to IFX, whose preoperative IFX and antibodies to IFX were tested within 60 days of surgery. This may represent a too broad range, making it difficult to draw strong conclusions. However, no difference in the overall infectious complication rate between patients with detectable serum levels and those with undetectable serum levels of IFX (cut-off value $1.4 \mu \mathrm{g} / \mathrm{ml}$ ) was shown. From these data, evidences to delay surgery in order to increase the time interval between the last dose of biologics and surgery seems to be relatively limited. (44)

Same results were reached by Zittan et al (36): no significant difference in the postoperative 2-stage IPAA leakage rate in UC patients operated on within 15 days, within 15 to 30 days, or 31 to 180 days from the last anti-TNF dose, nor were there differences based on the presence of detectable IFX serum levels (cut-off value $0.98 \mu \mathrm{g} / \mathrm{ml}$ ). A similar pattern of result was obtained by Lau et al from the analysis of 94 UC patients with anti-TNF (IFX and adalimumab) serum levels drawn 7 days preoperatively. There were no significant differences in adverse postoperative outcomes (overall and infectious complications) between the detectable and undetectable serum anti-TNF groups in the entire UC cohort or when patients were stratified according to type of surgery (first stage vs delayed pouch construction). In the CD cohort, there was a higher but statistically insignificant rate of adverse outcomes in the detectable group. Using a cut-off level of 3 $\mu \mathrm{g} / \mathrm{ml}$, postoperative morbidity $(\mathrm{OR}=2.5, \mathrm{p}=0.03$ ) and infectious complications $(\mathrm{OR}=3.0, \mathrm{p}=0.03$ ) were significantly higher in the $\geq 3 \mu \mathrm{g} / \mathrm{ml}$ group. There were higher rates of postoperative morbidity $(p=0.047)$ and hospital readmissions ( $p=0.04$ ) in the $\geq 8 \mu \mathrm{g} / \mathrm{ml}$ compared to $<3 \mu \mathrm{g} / \mathrm{ml}$ group. (43) These studies are largely underpowered and results and conclusions may suffer from other confounding factors such as mixed IBD population, different cut-offs for anti-TNF serum levels, different timepoint of assessment of the anti-TNF serum level, heterogeneity with regard to studies populations and index surgery.

The failure to demonstrate that high IFX serum levels put the patients at risk of postoperative complications, might be explained by inter-individual differences in drugs pharmacokinetics and immunogenicity that leads to variable response in clinical efficacy and, correspondingly, in the rate of the possible side effects. On this point it might be speculate that the cumulative dose of anti-TNF drugs might better reflect the risk of postoperative infections than the time interval alone. This for two reasons. First, the severity and the extent of the disease due to a larger surface of inflamed intestine could accelerate the drug clearance (45). Secondly, in the modern era of biologics use, patients who are referred to surgery are more prone to be exposed to different regimens of medications (steroids and immunomodulators among others) as result of the increased tendency to insist on medical therapy and to consider surgery as ultimate resort (step-up approach). In this regard, more and more data have proven a relation between combination of steroids and biologics with postoperative septic complications. $(35,46)$. As consequence of this, the cumulative dose of exposure to antiTNF might serves as indirect sign of more severe disease and sicker patients with compromitted general conditions and impaired nutritional status. Selvaggi et al in their meta-analysis showed, after subgroup analysis of IPAA-related complications including only good quality studies according to the STROBE score (47), that the risk difference of postoperative infectious complications between IFX and non-IFX patients was not related to the time interval between last dose administration and surgery but to the number of administrations received ( $\geq 3$ ). (39) Data on other anti-TNF agents (adalimumab and golimumab) are unfortunately largely absent.

\section{- Approach in clinical practice}

It is our convincement that severe active UC and prolonged exposure to anti-TNF is an absolute contraindication for the construction of a pouch during the first step of the surgical treatment. (27) Even if uniform evidence is lacking, what emerges from an attentive analysis of the literature and from the personal experience in a tertiary referral centrum for IBD is that performing a pouch simultaneously to a proctocolectomy altered the postoperative course of UC patients, increasing the risk for short- term infectious and non-infectious complications and for long-term pouch failure. $(36,48,49)$ In line with this, it is notable that since the introduction of biologics a larger proportion of patients undergoes a total abdominal 
colectomy as first index surgery for the treatment of refractory UC. (27) This trend is highlighted by large scale data derived from population-based studies in the United States $(50,51)$ and by single institution reports. (49) Geltzeimer et al demonstrated that an initial total abdominal colectomy has surpassed initial restorative proctocolectomy as the most common initial surgical intervention for UC since 2008 in the United States. In particular, initial total abdominal colectomy increases by 44\% between 1991 and 2011, while over the same period, initial restorative proctocolectomy decreased from 62 to $46 \%$ (51).

This shift in the clinical practice is supported by evidences derived from cohort and registry-based studies from Europe and North America. Kulayat et al, in a series of more than 2400 UC patients, clearly found a correlation between postoperative complications and IPAA after biologics exposure, when pouch construction was done as first step of surgery. By contrast, no detrimental effect of anti-TNF agents on complications in patients who underwent resection without anastomosis as their initial UC-related surgery was shown. (52) Similar results were retrieved from a study from Mayo clinic published in 2013 with a cohort of 588 patients, clearly stating that patients who received biologics prior to a one-stage procedure presented a 3-times risk of pelvic sepsis with a remarkable impact on long-term pouch function. (48) At the same conclusion came the most recent meta-analysis by Selvaggi et al. (39) showing that, in the setting of a 2-stage procedure, a diversion ileostomy does not prevent IPAA-related complications in the long term. Furthermore, other cohort studies indicate that the presence of a diversion ileostomy does not seem to mitigate early septic complications related to IPAA construction. $(29,30,39,48)$ Further studies failed to prove a detrimental effect on postoperative complications in patients undergoing IPAA as primary surgical step since the anti-TNF arm was not adequately powered to detect difference in complications rate. $(33,35,53,54)$

These findings suggest that the operative strategy modifies short and long-term outcome in patients with UC on preoperative anti-TNF therapy. If avoiding the IPAA formation as primary surgery for UC refractory patients seems to be well established (ref), it remains still unclear whether a diversion stoma should be use at the time of the construction of a pouch after delayed rest proctectomy. In other words, incertitude exists whether a 3-stage or a modified 2-stage approach would be more appropriate for this sort of patients. Adverse consequences of ileostomy are well established: patients managing their ileostomy put their life on hold during the interval until closure; closure mandates an extra operation with attendant hospital stay and well-described complications, including obstruction and anastomotic leak (55); as well as excess treatment costs of stoma appliances and ileostomy closure. A case series from the United States demonstrated that the pouch outcomes in case of a modified 2-stage procedure are comparable with the 3-stage procedure, with the advantage of having a shorter length of stay and reduced costs. (56) In another comparative study from Canada, patients who underwent a modified 2-stage procedure had a lower rate of anastomotic leak following IPAA. (57) We recently published a study to evaluate the outcomes of selective ileostomy formation in a multicenter cohort of 621 patients undergoing pouch surgery. Diverted patients showed more weight loss and had a higher ASA score. In the ileostomy group, more patients were treated with steroids and antiTNF. No different was found in the leakage rates between diverted and non-diverted patients. Moreover, defunctioning was associated with an increased risk of small bowel obstruction. (58)

On the basis of the recent evidence suggestion about the superiority of a modified 2-stage over the traditional 2-stage procedure in terms of rate of anastomotic leakage (57), and the risk of stoma-related complications (e.g. SBO) when a diversion stoma is made (58), it seems appropriate to choose for an initial subtotal colectomy with terminal ileostomy followed by a proctectomy with an IPAA without diversion ileostomy (modified 2 stage approach) in case of refractory UC patients previously exposed to anti-TNF drugs.

\section{Conclusions}

To sum up what emerged from this review: 
- Studies investigating the role of anti-TNF agents in increasing the rate of postoperative complications after pouch surgery are largely contradictory. In literature, three meta-analysis have been published with discrepancy in results and conclusions.

- Studies are mostly retrospective, monocentric, underpowered and strongly affected by bias with regard to type of surgery performed, patients selection, severity of disease, interval between last administration of anti-TNF agents and surgery, duration of exposure to anti-TNF agents, concomitant administration of other drugs (steroids and immunosuppressants), off-label use of IFX prior to 2005.

- Data on other anti-TNF agents such as adalimumab and golimumab are largely absent.

- A correlation between serum levels of IFX at the time of surgery and the occurrence of postoperative complications in patients with UC is not demonstrated.

- The cumulative dose of anti-TNF agents might better reflect the risk of postoperative infections than the time interval alone in patients undergoing pouch surgery, but probably only because of the association with more severe disease.

- The construction of a pouch as first step of surgery in refractory UC patients (one-stage or 2-stage proctocolectomy) with prolonged exposure to anti-TNF should be avoided. In this setting, a modified 2-stage or a 3-stage approach is preferable.

- A diversion ileostomy puts the patients at risk of stoma-related complications (SBO) without mitigating the risk for leakage. As consequence of this, a modified 2-stage might be preferred over a 3-stage procedure in patients preoperatively exposed to anti-TNF. This strategy seems to reduce the rate of anastomotic leakage and unnecessary stomas, improving short-term and long-term outcomes.

\begin{tabular}{|c|c|c|c|}
\hline & $1^{\text {th }}$ stage & $2^{\text {nd }}$ stage & $3^{\text {th }}$ stage \\
\hline 1-stage & $\begin{aligned} & \text { Proctocolectomy } \\
+ & \text { pouch construcion }\end{aligned}$ & & \\
\hline 2-stage & $\begin{array}{l}\text { Proctocolectomy } \\
\text { + pouch construction } \\
\text { + loop ileostomy }\end{array}$ & Closure of ileostomy & \\
\hline $\begin{array}{l}\text { modified } \\
\text { 2-stage }\end{array}$ & $\begin{array}{l}\text { Subtotal colectomy } \\
+ \text { end-ileostomy }\end{array}$ & $\begin{array}{l}\text { Completion proctectomy } \\
+ \text { pouch construction }\end{array}$ & \\
\hline 3-stage & $\begin{array}{l}\text { Subtotal colectomy } \\
\text { + end-ileostomy }\end{array}$ & $\begin{array}{l}\text { Completion proctectomy } \\
+ \text { pouch construction } \\
\text { + loop ileostomy }\end{array}$ & Closure of ileostomy \\
\hline
\end{tabular}

Table 1. Types of different surgical stages in the surgical treatment of UC.

IPAA: ileal pouch-anal anastomosis 


\section{References}

1. Cohen RD. The pharmacoeconomics of biologic therapy for IBD. Nat Rev Gastroenterol Hepatol. 2010;7(2):103-9.

2. Oresland T, Bemelman WA, Sampietro GM, Spinelli A, Windsor A, Ferrante M, et al. European evidence based consensus on surgery for ulcerative colitis. J Crohns Colitis. 2015;9(1):4-25.

3. Brown SR, Faiz, O.D. et al. lleoanal pouch report. Association of coloproctology of Ireland and Great Britain. 2017.

4. Langholz E, Munkholm P, Davidsen M, Binder V. Colorectal cancer risk and mortality in patients with ulcerative colitis. Gastroenterology. 1992;103(5):1444-51.

5. Annese V, Duricova D, Gower-Rousseau C, Jess T, Langholz E. Impact of New Treatments on Hospitalisation, Surgery, Infection, and Mortality in IBD: a Focus Paper by the Epidemiology Committee of ECCO. J Crohns Colitis. 2016;10(2):216-25.

6. Niewiadomski O, Studd C, Hair C, Wilson J, Ding NS, Heerasing N, et al. Prospective populationbased cohort of inflammatory bowel disease in the biologics era: Disease course and predictors of severity. J Gastroenterol Hepatol. 2015;30(9):1346-53.

7. Bernstein CN, Ng SC, Lakatos PL, Moum B, Loftus EV, Jr., Epidemiology, et al. A review of mortality and surgery in ulcerative colitis: milestones of the seriousness of the disease. Inflamm Bowel Dis. 2013;19(9):2001-10.

8. de Silva S, Devlin S, Panaccione R. Optimizing the safety of biologic therapy for IBD. Nat Rev Gastroenterol Hepatol. 2010;7(2):93-101.

9. Rutgeerts P, Sandborn WJ, Feagan BG, Reinisch W, Olson A, Johanns J, et al. Infliximab for induction and maintenance therapy for ulcerative colitis. N Engl J Med. 2005;353(23):2462-76.

10. Viget N, Vernier-Massouille G, Salmon-Ceron D, Yazdanpanah Y, Colombel JF. Opportunistic infections in patients with inflammatory bowel disease: prevention and diagnosis. Gut. 2008;57(4):549-58.

11. Toruner M, Loftus EV, Jr., Harmsen WS, Zinsmeister AR, Orenstein R, Sandborn WJ, et al. Risk factors for opportunistic infections in patients with inflammatory bowel disease. Gastroenterology. 2008;134(4):929-36.

12. Keane J, Gershon S, Wise RP, Mirabile-Levens E, Kasznica J, Schwieterman WD, et al. Tuberculosis associated with infliximab, a tumor necrosis factor alpha-neutralizing agent. N Engl J Med. 2001;345(15):1098-104.

13. Melville DM, Ritchie JK, Nicholls RJ, Hawley PR. Surgery for ulcerative colitis in the era of the pouch: the St Mark's Hospital experience. Gut. 1994;35(8):1076-80.

14. Fazio VW, Ziv Y, Church JM, Oakley JR, Lavery IC, Milsom JW, et al. Ileal pouch-anal anastomoses complications and function in 1005 patients. Ann Surg. 1995;222(2):120-7.

15. Ulich TR, del Castillo J, Keys M, Granger GA, Ni RX. Kinetics and mechanisms of recombinant human interleukin 1 and tumor necrosis factor-alpha-induced changes in circulating numbers of neutrophils and lymphocytes. J Immunol. 1987;139(10):3406-15.

16. Mooney DP, O'Reilly M, Gamelli RL. Tumor necrosis factor and wound healing. Ann Surg. 1990;211(2):124-9.

17. Fu X, Tian H, Hsu S, Wang D, Sheng Z. In vivo effects of tumor necrosis factor-alpha on incised wound and gunshot wound healing. J Trauma. 1996;40(3 Suppl):S140-3.

18. Salomon GD, Kasid A, Cromack DT, Director E, Talbot TL, Sank A, et al. The local effects of cachectin/tumor necrosis factor on wound healing. Ann Surg. 1991;214(2):175-80.

19. Rapala K, Laato M, Niinikoski J, Kujari H, Soder O, Mauviel A, et al. Tumor necrosis factor alpha inhibits wound healing in the rat. Eur Surg Res. 1991;23(5-6):261-8.

20. Baek SJ, Dozois EJ, Mathis KL, Lightner AL, Boostrom SY, Cima RR, et al. Safety, feasibility, and shortterm outcomes in 588 patients undergoing minimally invasive ileal pouch-anal anastomosis: a singleinstitution experience. Tech Coloproctol. 2016;20(6):369-74. 
21. Gallo G, Kotze PG, Spinelli A. Surgery in ulcerative colitis: When? How? Best Pract Res Clin Gastroenterol. 2018;32-33:71-8.

22. de Buck van Overstraeten A, Wolthuis AM, D'Hoore A. Transanal completion proctectomy after total colectomy and ileal pouch-anal anastomosis for ulcerative colitis: a modified single stapled technique. Colorectal Dis. 2016;18(4):0141-4.

23. McKenna NP, Dozois EJ, Pemberton JH, Lightner AL. Impact of sex on 30-day complications and long-term functional outcomes following ileal pouch-anal anastomosis for chronic ulcerative colitis. Int J Colorectal Dis. 2018;33(5):619-25.

24. Mark-Christensen A, Erichsen R, Brandsborg S, Pachler FR, Norager CB, Johansen N, et al. Pouch failures following ileal pouch-anal anastomosis for ulcerative colitis. Colorectal Dis. 2018;20(1):44-52.

25. Kiely JM, Fazio VW, Remzi FH, Shen B, Kiran RP. Pelvic sepsis after IPAA adversely affects function of the pouch and quality of life. Dis Colon Rectum. 2012;55(4):387-92.

26. Sagap I, Remzi FH, Hammel JP, Fazio VW. Factors associated with failure in managing pelvic sepsis after ileal pouch-anal anastomosis (IPAA)--a multivariate analysis. Surgery. 2006;140(4):691-703; discussion $-4$.

27. Holubar SD, Holder-Murray J, Flasar M, Lazarev M. Anti-Tumor Necrosis Factor-alpha Antibody Therapy Management Before and After Intestinal Surgery for Inflammatory Bowel Disease: A CCFA Position Paper. Inflamm Bowel Dis. 2015;21(11):2658-72.

28. Selvasekar CR, Cima RR, Larson DW, Dozois EJ, Harrington JR, Harmsen WS, et al. Effect of infliximab on short-term complications in patients undergoing operation for chronic ulcerative colitis. J Am Coll Surg. 2007;204(5):956-62; discussion 62-3.

29. Mor IJ, Vogel JD, da Luz Moreira A, Shen B, Hammel J, Remzi FH. Infliximab in ulcerative colitis is associated with an increased risk of postoperative complications after restorative proctocolectomy. Dis Colon Rectum. 2008;51(8):1202-7; discussion 7-10.

30. Eshuis EJ, Al Saady RL, Stokkers PC, Ponsioen CY, Tanis PJ, Bemelman WA. Previous infliximab therapy and postoperative complications after proctocolectomy with ileum pouch anal anastomosis. J Crohns Colitis. 2013;7(2):142-9.

31. Krane MK, Allaix ME, Zoccali M, Umanskiy K, Rubin MA, Villa A, et al. Preoperative infliximab therapy does not increase morbidity and mortality after laparoscopic resection for inflammatory bowel disease. Dis Colon Rectum. 2013;56(4):449-57.

32. Norgard BM, Nielsen J, Qvist N, Gradel KO, de Muckadell OB, Kjeldsen J. Pre-operative use of antiTNF-alpha agents and the risk of post-operative complications in patients with ulcerative colitis - a nationwide cohort study. Aliment Pharmacol Ther. 2012;35(11):1301-9.

33. Schluender SJ, Ippoliti A, Dubinsky M, Vasiliauskas EA, Papadakis KA, Mei L, et al. Does infliximab influence surgical morbidity of ileal pouch-anal anastomosis in patients with ulcerative colitis? Dis Colon Rectum. 2007;50(11):1747-53.

34. Kunitake H, Hodin R, Shellito PC, Sands BE, Korzenik J, Bordeianou L. Perioperative treatment with infliximab in patients with Crohn's disease and ulcerative colitis is not associated with an increased rate of postoperative complications. J Gastrointest Surg. 2008;12(10):1730-6; discussion 6-7.

35. Ferrante M, D'Hoore A, Vermeire S, Declerck S, Noman M, Van Assche G, et al. Corticosteroids but not infliximab increase short-term postoperative infectious complications in patients with ulcerative colitis. Inflamm Bowel Dis. 2009;15(7):1062-70.

36. Zittan E, Milgrom R, Ma GW, Wong-Chong N, O'Connor B, McLeod RS, et al. Preoperative Antitumor Necrosis Factor Therapy in Patients with Ulcerative Colitis Is Not Associated with an Increased Risk of Infectious and Noninfectious Complications After Ileal Pouch-anal Anastomosis. Inflamm Bowel Dis. 2016;22(10):2442-7.

37. Yang Z, Wu Q, Wu K, Fan D. Meta-analysis: pre-operative infliximab treatment and short-term postoperative complications in patients with ulcerative colitis. Aliment Pharmacol Ther. 2010;31(4):486-92.

38. Yang Z, Wu Q, Wang F, Wu K, Fan D. Meta-analysis: effect of preoperative infliximab use on early postoperative complications in patients with ulcerative colitis undergoing abdominal surgery. Aliment Pharmacol Ther. 2012;36(10):922-8. 
39. Selvaggi F, Pellino G, Canonico S, Sciaudone G. Effect of preoperative biologic drugs on complications and function after restorative proctocolectomy with primary ileal pouch formation: systematic review and meta-analysis. Inflamm Bowel Dis. 2015;21(1):79-92.

40. Sandborn WJ, Hanauer SB. Antitumor necrosis factor therapy for inflammatory bowel disease: a review of agents, pharmacology, clinical results, and safety. Inflamm Bowel Dis. 1999;5(2):119-33.

41. Klotz U, Teml A, Schwab M. Clinical pharmacokinetics and use of infliximab. Clin Pharmacokinet. 2007;46(8):645-60.

42. Ternant D, Aubourg A, Magdelaine-Beuzelin C, Degenne D, Watier H, Picon L, et al. Infliximab pharmacokinetics in inflammatory bowel disease patients. Ther Drug Monit. 2008;30(4):523-9.

43. Lau C, Dubinsky M, Melmed G, Vasiliauskas E, Berel D, McGovern D, et al. The impact of preoperative serum anti-TNFalpha therapy levels on early postoperative outcomes in inflammatory bowel disease surgery. Ann Surg. 2015;261(3):487-96.

44. Waterman M, Xu W, Dinani A, Steinhart AH, Croitoru K, Nguyen GC, et al. Preoperative biological therapy and short-term outcomes of abdominal surgery in patients with inflammatory bowel disease. Gut. 2013;62(3):387-94.

45. Ordas I, Feagan BG, Sandborn WJ. Therapeutic drug monitoring of tumor necrosis factor antagonists in inflammatory bowel disease. Clin Gastroenterol Hepatol. 2012;10(10):1079-87; quiz e85-6.

46. Magro F, Gionchetti P, Eliakim R, Ardizzone S, Armuzzi A, Barreiro-de Acosta M, et al. Third European Evidence-based Consensus on Diagnosis and Management of Ulcerative Colitis. Part 1: Definitions, Diagnosis, Extra-intestinal Manifestations, Pregnancy, Cancer Surveillance, Surgery, and lleoanal Pouch Disorders. J Crohns Colitis. 2017;11(6):649-70.

47. von Elm E, Altman DG, Egger M, Pocock SJ, Gotzsche PC, Vandenbroucke JP, et al. The Strengthening the Reporting of Observational Studies in Epidemiology (STROBE) statement: guidelines for reporting observational studies. J Clin Epidemiol. 2008;61(4):344-9.

48. Gu J, Remzi FH, Shen B, Vogel JD, Kiran RP. Operative strategy modifies risk of pouch-related outcomes in patients with ulcerative colitis on preoperative anti-tumor necrosis factor-alpha therapy. Dis Colon Rectum. 2013;56(11):1243-52.

49. Gu J, Stocchi L, Ashburn J, Remzi FH. Total abdominal colectomy vs. restorative total proctocolectomy as the initial approach to medically refractory ulcerative colitis. Int J Colorectal Dis. 2017;32(8):1215-22.

50. Bikhchandani J, Polites SF, Wagie AE, Habermann EB, Cima RR. National trends of 3-versus 2-stage restorative proctocolectomy for chronic ulcerative colitis. Dis Colon Rectum. 2015;58(2):199-204.

51. Geltzeiler CB, Lu KC, Diggs BS, Deveney KE, Keyashian K, Herzig DO, et al. Initial surgical management of ulcerative colitis in the biologic era. Dis Colon Rectum. 2014;57(12):1358-63.

52. Kulaylat AS, Kulaylat AN, Schaefer EW, Tinsley A, Williams E, Koltun W, et al. Association of Preoperative Anti-Tumor Necrosis Factor Therapy With Adverse Postoperative Outcomes in Patients Undergoing Abdominal Surgery for Ulcerative Colitis. JAMA Surg. 2017;152(8):e171538.

53. Coquet-Reinier B, Berdah SV, Grimaud JC, Birnbaum D, Cougard PA, Barthet M, et al. Preoperative infliximab treatment and postoperative complications after laparoscopic restorative proctocolectomy with ileal pouch-anal anastomosis: a case-matched study. Surg Endosc. 2010;24(8):1866-71.

54. Gainsbury ML, Chu DI, Howard LA, Coukos JA, Farraye FA, Stucchi AF, et al. Preoperative infliximab is not associated with an increased risk of short-term postoperative complications after restorative proctocolectomy and ileal pouch-anal anastomosis. J Gastrointest Surg. 2011;15(3):397-403.

55. Carlsson E, Fingren J, Hallen AM, Petersen C, Lindholm E. The Prevalence of Ostomy-related Complications 1 Year After Ostomy Surgery: A Prospective, Descriptive, Clinical Study. Ostomy Wound Manage. 2016;62(10):34-48.

56. Swenson BR, Hollenbeak CS, Poritz LS, Koltun WA. Modified two-stage ileal pouch-anal anastomosis: equivalent outcomes with less resource utilization. Dis Colon Rectum. 2005;48(2):256-61. 57. Zittan E, Wong-Chong N, Ma GW, McLeod RS, Silverberg MS, Cohen Z. Modified Two-stage Ileal Pouch-Anal Anastomosis Results in Lower Rate of Anastomotic Leak Compared with Traditional Two-stage Surgery for Ulcerative Colitis. J Crohns Colitis. 2016;10(7):766-72. 
58. Sahami S, Buskens CJ, Fadok TY, Tanis PJ, de Buck van Overstraeten A, Wolthuis AM, et al. Defunctioning lleostomy is not Associated with Reduced Leakage in Proctocolectomy and Ileal Pouch Anastomosis Surgeries for IBD. J Crohns Colitis. 2016;10(7):779-85. 